\title{
Queuing and Statistical Analysis of Freeway Bottleneck Formation
}

\author{
Shantanu Das ${ }^{1}$ and David Levinson ${ }^{2}$
}

\begin{abstract}
A modified approach to treat traffic flow parameters (flow, density, and speed) has been introduced in this paper. A queuing analysis has been conducted on traffic flow data on Interstate 94 in the Minneapolis-St. Paul metro area. A methodology has been developed to calibrate loop detector count data. Corrected flow data has been subjected to analysis using queuing analysis to compute densities and speeds on freeway sections. Statistical analysis identifies "active bottleneck" locations on freeways and sections where bottlenecks occur because of disturbances caused by downstream bottlenecks propagating backwards in the form of shockwaves. A sample of 6 days on Interstate 94 was considered for the analysis. Our analysis reveals that the same section cannot always be characterized as a "bottleneck" location; at some times it is active and at others it is subject to downstream bottlenecks. Traffic flow characteristics change and that leads to changing situations on each freeway section.
\end{abstract}

DOI: 10.1061/(ASCE)0733-947X(2004)130:6(787)

CE Database subject headings: Traffic management; Statistical analysis; Data processing; Error analysis; Traffic congestion; Traffic flow.

\section{Introduction}

This paper has its roots in the Ramp Meter shut-off experiment that was conducted in the Twin Cities of Minneapolis and St. Paul during the fall of 2000. Along with determining the effectiveness of ramp metering in controlling freeway traffic, this experiment provided an opportunity to study traffic flow characteristics under both metered and unmetered conditions. This was made possible by the availability of a considerable amount of high quality loop detector data collected both during the period preceding the experiment and during the experiment. Our research applies queuing analysis to traffic data collected from loop detectors during unmetered conditions to determine the location of bottlenecks. It thus extends previous research by Cassidy and others (Cassidy 2001; Cassidy and Bertini 2001; Cassidy et al. 2002).

The paper also attempts to provide an alternate way of predicting active bottlenecks using queuing and statistical analyses. The importance of accurately predicting active bottlenecks can be hardly overemphasized. Recent studies by Agyemang-Duah and Hall (1991) show how determination of freeway capacity hinges on the location of active bottleneck. Active bottlenecks show traits of not being affected by traffic conditions downstream (Daganzo 1997) and that once an active bottleneck is formed, both

\footnotetext{
${ }^{1}$ Transportation Analyst, Wilbur Smith Associates, 900 Chapel St., New Haven, CT 06510. E-mail: sdas@wilbursmith.com

${ }^{2}$ Assistant Professor, Dept. of Civil Engineering, Univ. of Minnesota, 500 Pillsbury Dr. SE, Minneapolis, MN (correspondence author). E-mail: levin031@umn.edu

Note. Discussion open until April 1, 2005. Separate discussions must be submitted for individual papers. To extend the closing date by one month, a written request must be filed with the ASCE Managing Editor. The manuscript for this paper was submitted for review and possible publication on December 9, 2002; approved on October 27, 2003. This paper is part of the Journal of Transportation Engineering, Vol. 130, No. 6, November 1, 2004. CASCE, ISSN 0733-947X/2004/6-787-795/ $\$ 18.00$.
}

"rabbits and slugs" involved in the bottleneck move at the same low speed with a drastic drop in queue discharge rate (Cassidy and Chung 2002). Active bottlenecks can be predicted based on real-time analysis of data generated by loop detectors (Bertini 2003).

Inductive loop detectors (ILD)s typically measure flow (the number of vehicles that pass it in some time period) and occupancy (the percentage of time for which the ILD is occupied in that time period). But the data provided by such ILDs are often fraught with errors. ILDs undercount or overcount depending on freeway traffic conditions. Moreover, the accuracy and consistency of detector data depend strongly on their installation and calibration procedures. A loop detector with percentage accuracy within 5\% is considered a "good" one (MDOT 2002). For a freeway with a daily output in the range of tens of thousands of vehicles, the 5\% error can amount to hundreds or thousands of vehicles per day. In addition, an underlying assumption required to measure speed when only single loop detectors are available is that effective vehicle lengths are uniform (Jia et al. 2001). This research presents a queuing analysis of freeway traffic that does not rely on effective vehicle lengths following earlier theory (Lawson et al. 1997).

The next section describes the data used in the analysis and the method for balancing counts across detector stations. Then queuing analysis is linked with the fundamental diagram of traffic flow. A typology of traffic conditions and rules for detecting active bottlenecks are provided. Then our results are presented, which apply the rules to identify active bottlenecks, sections that are subject to downstream bottlenecks, and free-flowing sections. The paper concludes by noting that at different times, the same section may be either an active bottleneck or subject to one, consistent with the definition provided by Daganzo (1997).

\section{Data}

The data used consisted primarily of 30-s flows and occupancies on freeways, aggregated to 5-min periods, to be consistent with 


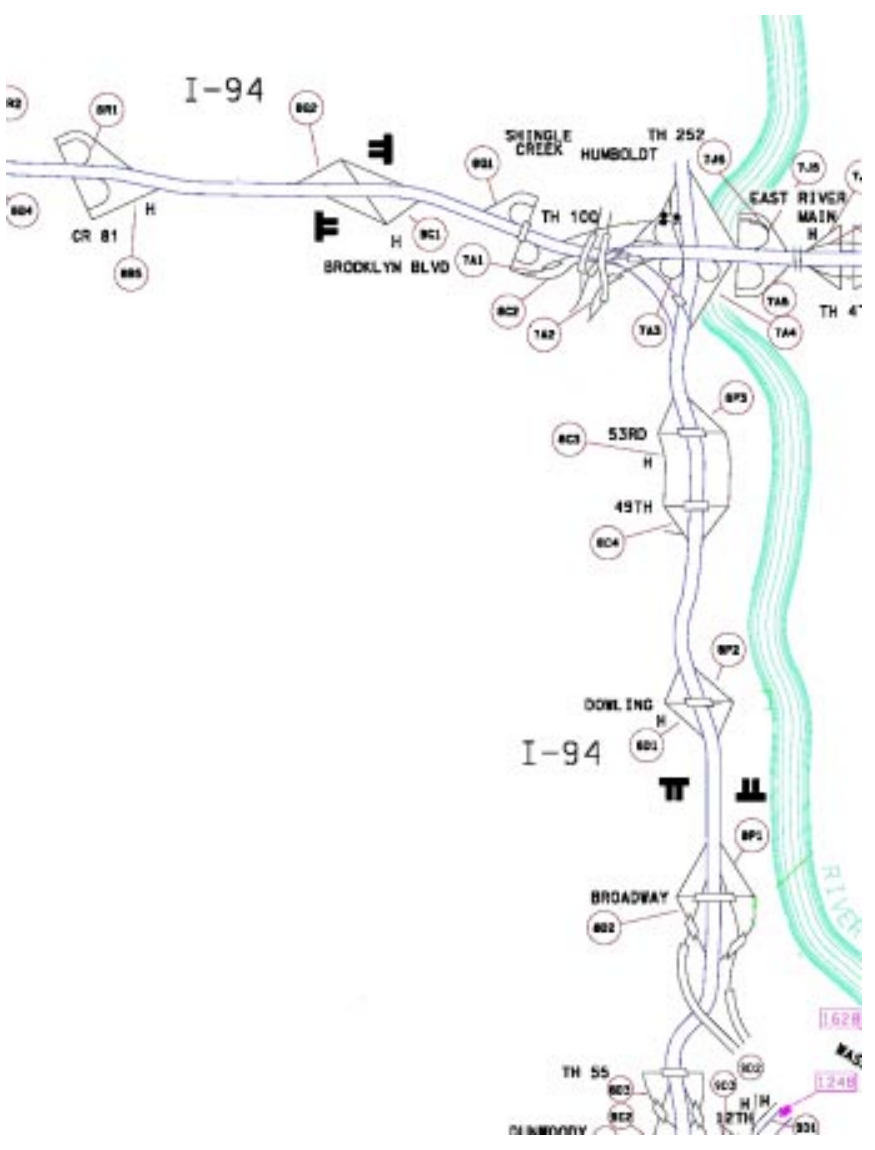

Fig. 1. Study locations

data from ramp detectors (which were only stored in 5-min intervals), collected by the loop detectors on Interstate 94 (between County Road 152 and Plymouth Avenue). The section is shown in Fig. 1. All the data were collected between November 1, 2000 and November 8, 2000, part of the period during which the ramp meters on the Twin Cities freeway network were shut-off. We need to ensure that we are dealing with good, reliable data. The methodology to ensure balanced flows compares the flows that entered and exited the freeway during some long time period (here $24 \mathrm{~h}$ ). The data is obtained for a carefully selected freeway section, which affords working detectors and which is long enough to be able to offer the possibility of observing bottleneck formation. Obvious detector errors or communication errors have already been eliminated. Comparison between the cumulative counts of all vehicles that went past the starting and ending points of the freeway section over a long period of time, $24 \mathrm{~h}$ in the analyses carried out here, gives the extent of detector error. To minimize the occurrence of existing vehicles on the roads, we begin at 3:30 a.m. when the freeway sections would be closest to being empty. While controlling for the on-ramps and off-ramps over a freeway section, we check if flow is conserved.

Over a long period of time

$$
\sum Q_{f_{\text {in }}}+\sum_{i}^{I} Q_{r_{\text {on }}}=\sum Q_{f_{\text {out }}}+\sum_{j}^{J} Q_{r_{\text {off }}}
$$

where the left-hand side (LHS) is the sum of the flows occurring over all the $I$ on-ramps $\left(Q_{r_{\text {on }}}\right)$ and the upstream freeway section $\left(Q_{f_{\text {in }}}\right)$ and the right-hand side (RHS) is the sum of the flows occurring over all the $J$ off-ramps $\left(Q_{r_{\text {off }}}\right)$ and the downstream

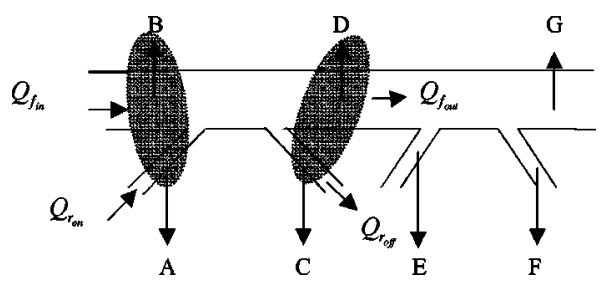

Fig. 2. Typical freeway section with upstream and downstream detectors

freeway section $\left(Q_{f_{\text {out }}}\right)$. This is illustrated in Fig. 2 .

Ensuring conservation of flow is an important step in transportation analysis (Barbour and Fricker 1990). Our next step is to clean the data such that the flows across various sections of the freeway balance over a long period of time $(24 \mathrm{~h})$. We identify a section on the freeway where the differences between inflows and outflows were at a minimum. This region would have around it a pair of detector stations that were defective to the least extent or not defective at all. Such a pair would be called a "least-error section." (Note that the detector station on the freeway may be a combination of two or more individual detectors.) This section was found after analyzing the data for $24 \mathrm{~h}$ on each of the 6 days that we analyzed the data from and its eligibility of being the section with "least error" was consistent across these 6 days. In the case when least-error section does not balance accurately as in the conservation equation (above), it was calibrated by allocating the errors into individual detector stations involved in the leasterror section in proportion to their flow.

Once the least-error section is identified and corrected, the adjustments in the total daily flows are done over successive detector stations both upstream and downstream of the least-error section. The adjoining detector stations were calibrated based on the value of the common detector station count (from the leasterror section) involved in the next section. For example, in Fig. 2, the ovals show the least-error section and the letters show the Detector Stations. A, B, C, and D, being a part of this least-error section, are each true and so when we go downstream, $\mathrm{G}+\mathrm{F}-\mathrm{E}$ should equal $\mathrm{D}$. This equality does not hold if there are errors in the final total counts on G, F, or E. These errors are eliminated by allocating the error to each of the Detector Stations G, F, and E weighted by their flows.

Mathematically, the correction for any detector station, A can be represented as

$$
C_{X}=\frac{Q_{X}}{\sum_{X=E, F, G} Q_{X}} \times\left|Q_{f_{\text {out }}}-Q_{f_{\text {in }}}+\sum_{1}^{j} Q_{r_{\text {off }}}-\sum_{1}^{i} Q_{r_{\text {on }}}\right|
$$

So

$$
\hat{Q}_{X}=Q_{X} \pm C_{X}
$$

where $\hat{Q}_{f_{\text {in }}}=$ corrected upstream freeway flow. $X=\mathrm{E}, \mathrm{F}$, and $\mathrm{G}$ referring to the regions in Fig. 2.

Note that there are $n$ detectors, $i$ on-ramps, and $j$ off-ramps involved in the section. We either add or subtract the correction depending on the need to increase or decrease the counts.

This process is continued farther downstream until we reach the end of the entire freeway section considered. The same procedure is also carried out in the upstream direction. The procedure described above is shown in the flowchart in Fig. 3. A computer program was coded in $C++$ to automate the above analysis. 


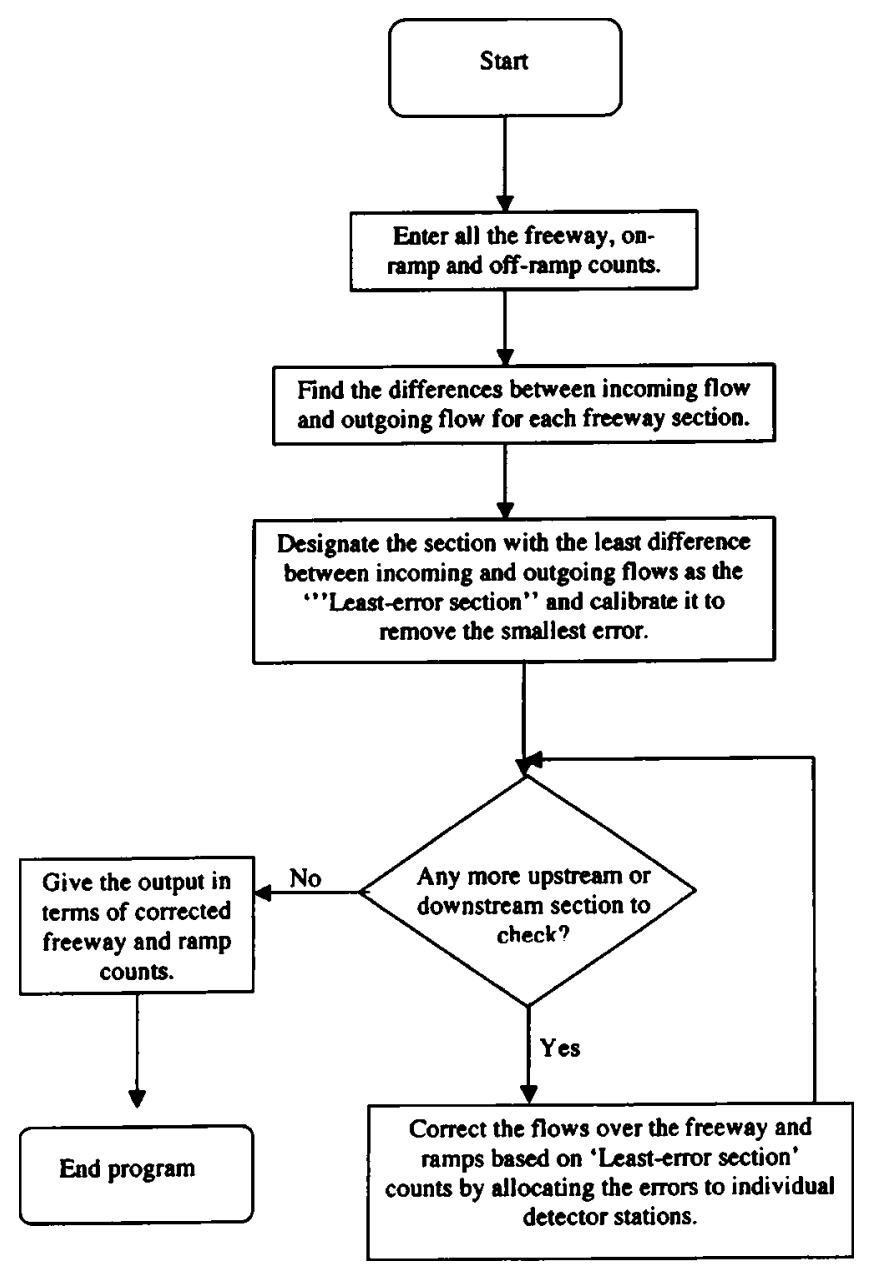

Fig. 3. Flowchart depicting count balancing process

After the corrected flows are obtained, the differences between the original counts and the new corrected counts are allocated to the individual detectors again according to the original flow proportions and to each $5 \mathrm{~min}$ time period. Mathematically,

$$
C_{a t}=\frac{Q_{a t}}{Q_{A}} \times C_{A}
$$

where $Q_{a}=$ cumulative $24 \mathrm{~h}$ flow over detector a; $Q_{A}=$ cumulative (24 h) flow over detector station, A, which includes all a detectors; $C_{A}=$ correction needed for station, $\mathrm{A}$; and $C_{a t}=$ correction needed in detector a count at time $t$.

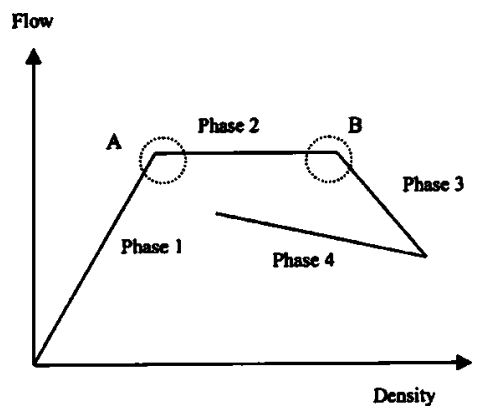

Fig. 4. Traffic phase diagram

\section{Quantification of Error Reduction}

The reduction in errors in detector counts is illustrated in Table 1, where six freeway sections are laid out and the differences in daily and mean hourly flows are calculated. We observe that the cumulative daily differences between flows coming into any freeway section and flows going out of the same freeway section almost vanishes. There are also large percentage reductions in mean hourly flow differences between input and output flows for each freeway section. We expect differences in hourly input and output flows as long as there are vehicles stored on the freeway section.

\section{Theory and Methodology}

It is hypothesized that if freeway traffic is allowed to behave without any restrictions imposed by ramp meters, it displays four phases in the flow-density curve as shown in Fig. 4.

- Phase 1 is the uncongested phase when there is no influence of the increasing density on the speeds of the vehicles. The speed does not drop with the introduction of newer vehicles onto the freeway.

- Phase 2 finds that the freeway cannot sustain the speed with injection of newer vehicles into the traffic stream. The density increases while speed falls, maintaining the flow.

- Phase 3 shows decreased speed and decreased flows. Very low speeds cause the queue discharge to drop at an active bottleneck, or a queue from a downstream bottleneck may be constraining the flow.

- Phase 4 is the recovery phase. During this phase, the density of traffic starts decreasing and speed starts increasing.

The circled region, A (in Fig. 4) is typically where we start observing "freeway breakdown." In other words, this region occurs when flow exceeds some critical capacity at a specific point

Table 1. Correction of Flows on Individual Freeway Sections

\begin{tabular}{|c|c|c|c|c|c|}
\hline Interstate 94 & Dai & & Mean & ices & \\
\hline Section between & Before & After & Before & After & reduction \\
\hline CR 152 and Xerxes Avenue & 369 & 1 & 21 & 2.12 & 89.90 \\
\hline Xerxes Avenue and Shingle Creek Parkway & 319 & 1 & 19.63 & 3.30 & 83.16 \\
\hline Shingle Creek Parkway and Dupont Avenue & 35 & 2 & 5.75 & 4.05 & 29.50 \\
\hline I-694 and 57th Avenue & 38 & 1 & 9.25 & 6.71 & 27.47 \\
\hline 57th Avenue and 49th Avenue & 52 & 1 & 4.75 & 1.41 & 70.25 \\
\hline 49th Avenue and 42nd Avenue & 30 & 1 & 6 & 4.08 & 31.97 \\
\hline
\end{tabular}

Note: Daily differences do not reduce to zero due to rounding error. 
and there is a drop in speed. However, detectors may have a lag in identifying this threshold as they may be upstream or downstream of the critical point. In the circled region, B, flow drops are associated with very low speeds. The phenomenon is particularly evidenced by the formation of queues upstream of where the breakdown occurs and a low discharge rate of vehicles due to sustained low speeds. Upstream detector flows are governed by downstream bottlenecks if there are long queues.

To perform the queuing analysis, we need a way of identifying the occurrence and location of bottlenecks. This requires identification of bottlenecks forming over space and time on the freeway.

Conventional traffic flow analyses have used flow and occupancy data that are provided by freeway detectors. From measured occupancy $(O)$, applying the following empirical formula gives density $(K)$ :

$$
K=\frac{O}{L_{\text {eff }}}
$$

where $L_{\text {eff }}=$ effective length of the average vehicle, that is the length of the vehicle from the front to the end plus the length of the detector.

Occupancy estimates the percentage of time a detector was occupied by vehicles within a certain time span and there is no way to ascertain the integrity of a detector by looking at the occupancy of that detector and detectors upstream or downstream of it. Whereas, "flow," the other parameter generated by detectors, allows us a way to check for detector errors using flow conservation as previously described. Moreover, the conventional density calculation assumes average "vehicle length" that may not be true.

An advantage of applying queuing analysis to traffic flow is that we do not have to rely on occupancy data obtained from individual detectors. Rather, we can find the densities directly from the corrected flows. Assuming an empty section (starting in the middle of the night), we count each car that passes the start and end of the section. At time $t$ we find the number of cars that are present on a freeway section by subtracting the cumulative number of cars that have passed the end of the freeway section

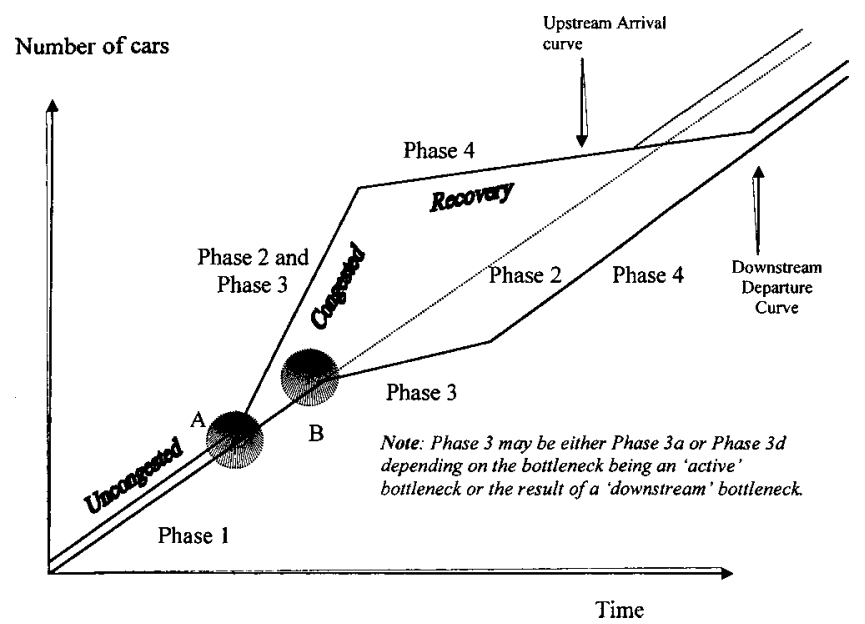

Fig. 5. Queuing input-output diagram: Relationship with phases

from the cumulative number of cars that passed the start of the freeway section. Mathematically

$$
N=\sum_{0}^{t} Q_{f_{\text {in }}}+\sum_{0}^{t} Q_{r_{\text {on }}}-\sum_{0}^{t} Q_{f_{\text {out }}}-\sum_{0}^{t} Q_{r_{\text {off }}}
$$

where $N=$ number of cars in the freeway section after a time $t$ from the start of counting. The normalized density $\left(K_{n}\right)$ in the section is given by

$$
K_{n}=\frac{N}{n \cdot L}
$$

where $L=$ length of the section; and $n=$ number of lanes in the section.

We repeatedly accumulate the vehicle flows over freeway sections. This leads to the drawback of applying queuing analysis to traffic flow, namely, errors tend to accumulate. However, starting with a clean set of data, and using the queuing diagram to reveal excess accumulation, ensures that such errors are small.

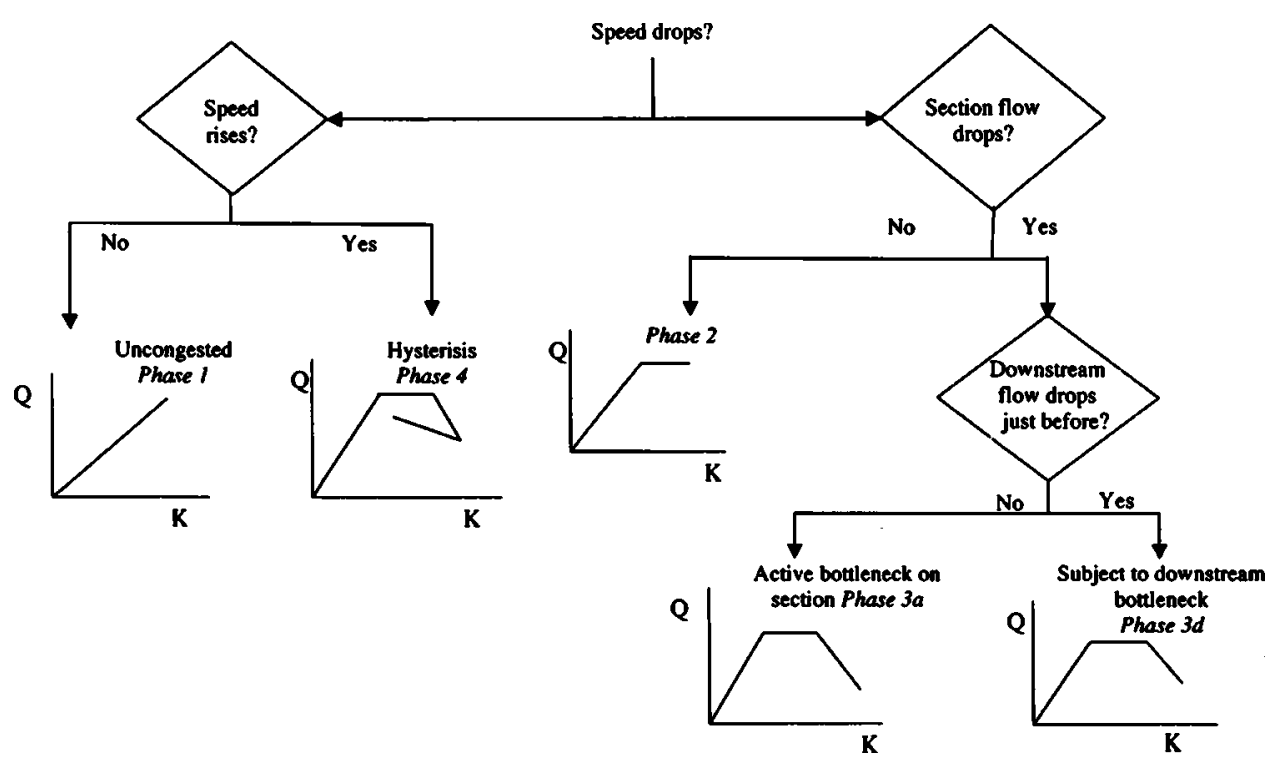

Fig. 6. Active bottleneck identification typology 


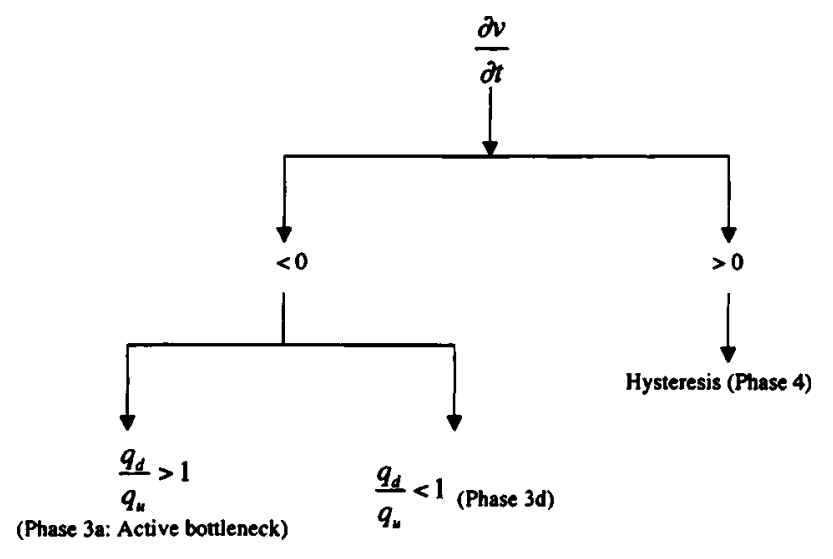

Fig. 7. Identification of phases

\section{Relationship between Queuing Diagram and Fundamental Diagram of Traffic Flow}

Looking at Fig. 4, we see that while cars move at free-flow speed in Phase 1, the density increases but flow remains uncongested. After a critical density is reached (Point A), we move over to Phase 2, speeds drop but flow does not. When we move over to Phase 3 (Point B), both speed and flow drop and bottleneck formation takes place. This bottleneck may be a result of a downstream bottleneck or the section may itself be the active bottleneck. Last comes Phase 4 wherein speeds recover and hysteresis takes place.

These relationships can also be observed with respect to the queuing diagram as shown in Fig. 5. It is easy to understand how the queuing diagram can explain the occurrence of phases. So long as the arrival rate is less than the service rate, the queue is uncongested, which is Phase 1. Congestion takes place when density increases beyond the critical density and that is when speed drops and bottleneck formation may also take place representing Phase 3. Hysteresis (Phase 4) is seen when speed recovers and
Table 2. Regression Results (Speed over Density) for Detector 52 (Broadway Avenue) on I-94

\begin{tabular}{lccc}
\hline & \multicolumn{2}{c}{ Dependent variable: speed } & \\
\cline { 2 - 3 } Variable & Hypothesis & Coefficient & $P>|Z|$ \\
\hline Constant & $+\mathrm{S}$ & 47.89 & $1.96 \mathrm{E}-14$ \\
Density & $\mathrm{NS}$ & 0.862 & 0.58 \\
\hline Note: Significance & based on & $90 \%$ & confidence \\
observations $=43$; and $R$ squared $=0.0075$.
\end{tabular}

the queue dissipates. Important to note are the different arrival and departure curves. It has been claimed earlier that speed drops at the bottleneck to such an extent that the bottleneck cannot sustain the maximum departure rate. That shows up in the departure curve that drops. However, this is only true in case of bottleneck formation at the section or downstream (Phase $3 a$ and Phase $3 d$, respectively). If it is Phase 2, then the departure curve remains the same (shown by a dotted line). Following this is the hysteresis in Phase 4. Also note the separation between departure and arrival curves even before the start of Phase 2 and after Phase 4, which reflects the vehicles that are already present in the section prior to and after the effects of queuing are over. The lateral shift in the departure curve occurs because once flow falls, it can only go back to the original departure rate and not beyond it. If bottleneck formation does not take place in the departure region, the arrival curve does not have to "recover" as much as it has to otherwise. The dotted line in the Phase 4 region again shows this.

\section{Active Bottleneck Identification}

We apply a systematic procedure in order to identify active bottlenecks. This framework is illustrated in Fig. 6.

The following steps describe the process of finding active bottlenecks:

1. Obtain the speeds over detectors from the flow-density-speed relationship $\left(V=Q / K_{n}\right)$, with flow and density calculated from queuing analysis.

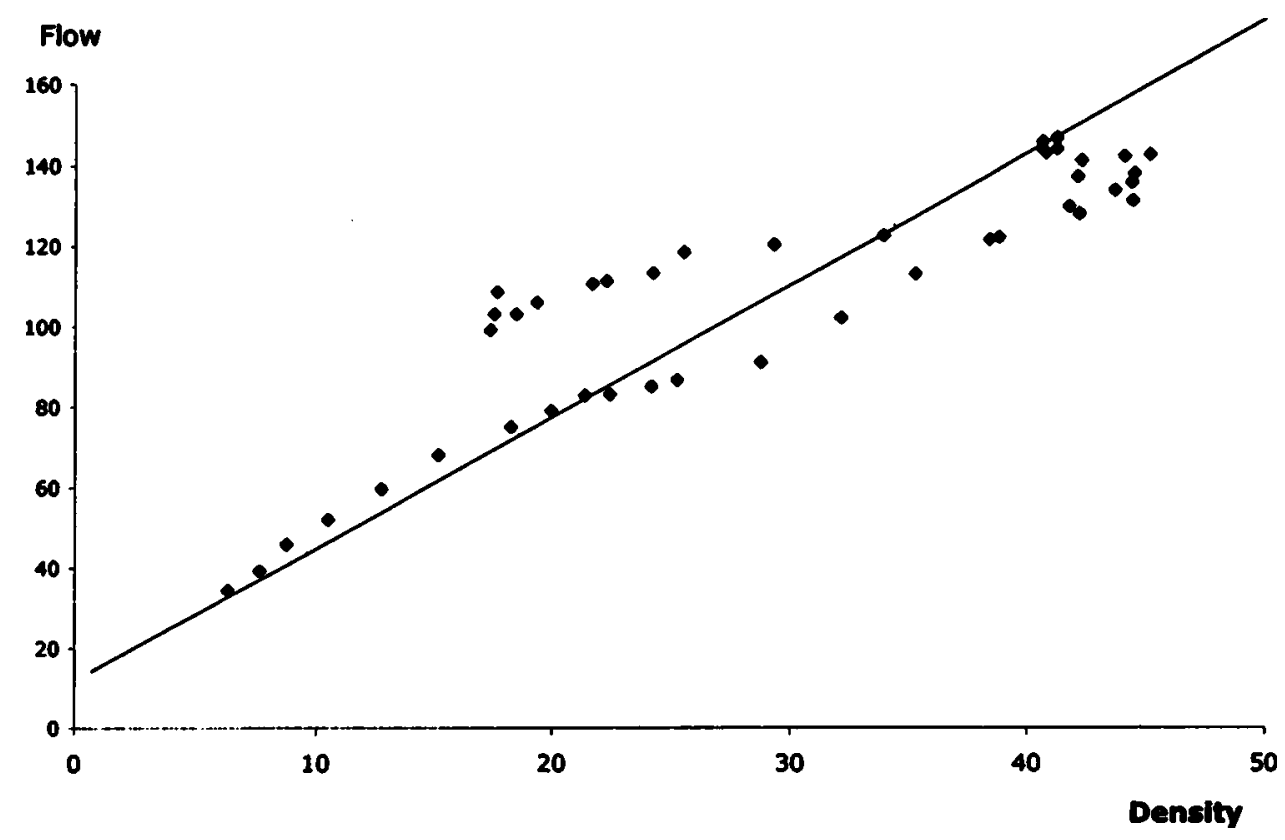

Fig. 8. Flow-density relationship for 52 (Lowry Avenue) 
2. Calculate five-interval moving averages of flow, density, and speed, each interval being 5 min long. This is done to smooth the transitions of each traffic flow characteristic.

3. Select a representative detector out of any station. However, consistency was maintained by selecting a middle lane detector. The subsequent detectors downstream would be selected from the same lane.

4. Select two periods to analyze: morning peak (beginning at 5:30 a.m. and continuing until 10:30 a.m.) and evening peak period (beginning at 1:30 p.m. and continuing until 6 p.m.). During the intermediate periods, data indicate that no queuing is taking place. Activity patterns are seen most distinctly during the morning and afternoon peaks.

5. Conduct statistical analysis of flow, density, and speed relationships to identify active bottlenecks for each selected detector.

6. Plot flows and speeds over time for each successive detector going upstream to downstream to support the results of the statistical analyses.

\section{Statistical Determination of Active Bottleneck Formation}

The typology developed in Fig. 6 has been implemented statistically and is detailed here.

\section{Phase 1}

Neither flow nor speed drops on a freeway section as density increases. Vehicles move at free-flow speeds.

$$
\frac{\partial V_{a}}{\partial K_{a}}=0 \pm \varepsilon_{1}
$$

To operationalize this, we regress speed as a function of density and test the significance of density. Statistically it is represented as
Table 3. Regression Results (Flow over Density, for Speeds below FreeFlow Speed) for Detector 83, near 42nd Avenue on I-94 on November 2, 2000

\begin{tabular}{lccc}
\hline & \multicolumn{2}{c}{ Dependent variable: flow } & \\
\cline { 2 - 3 } Variable & Hypothesis & Coefficient & $P>|Z|$ \\
\hline Constant & $+\mathrm{S}$ & 68.23 & $1.96 \mathrm{E}-14$ \\
Density & $\mathrm{NS}$ & 0.127 & 0.58 \\
\hline
\end{tabular}

Note: Significance based on $90 \%$ confidence interval; number of observations $=49$; and $R$ squared $=0.011$.

$$
V_{a}=\alpha_{0}+\alpha_{1} \cdot K_{a}
$$

where $\varepsilon_{1}=$ allowable error; $V_{a}=$ speed over detector $a$; and $K_{a}=$ density over detector $a$.

If $\alpha_{1}$ is not statistically different from zero, we conclude that this is the uncongested Phase 1 . Note as the number of observations increases, the likelihood of statistical significance increases, thus our test must get more rigorous.

Phase 2

Speed drops on a section but flow does not. In this case, flows are maintained with occurrence of high densities but low speeds.

$$
\frac{\partial Q_{a}}{\partial K_{a}}=0 \pm \varepsilon_{2}
$$

From the analysis of change in speed above, we conclude that it is not Phase 1. To operationalize this measure, for such a detector, we separate the time intervals when speed drops by more than one standard deviation from the average speed over the whole period and regress the flows over densities for such time intervals. Statistically

$$
Q_{a}=\beta_{0}+\beta_{1} \cdot K_{a}
$$

where $Q_{a}=$ flow over detector $a$, and $K_{a}=$ density over detector $a$. And we test for statistical significance of $\beta_{1}$.

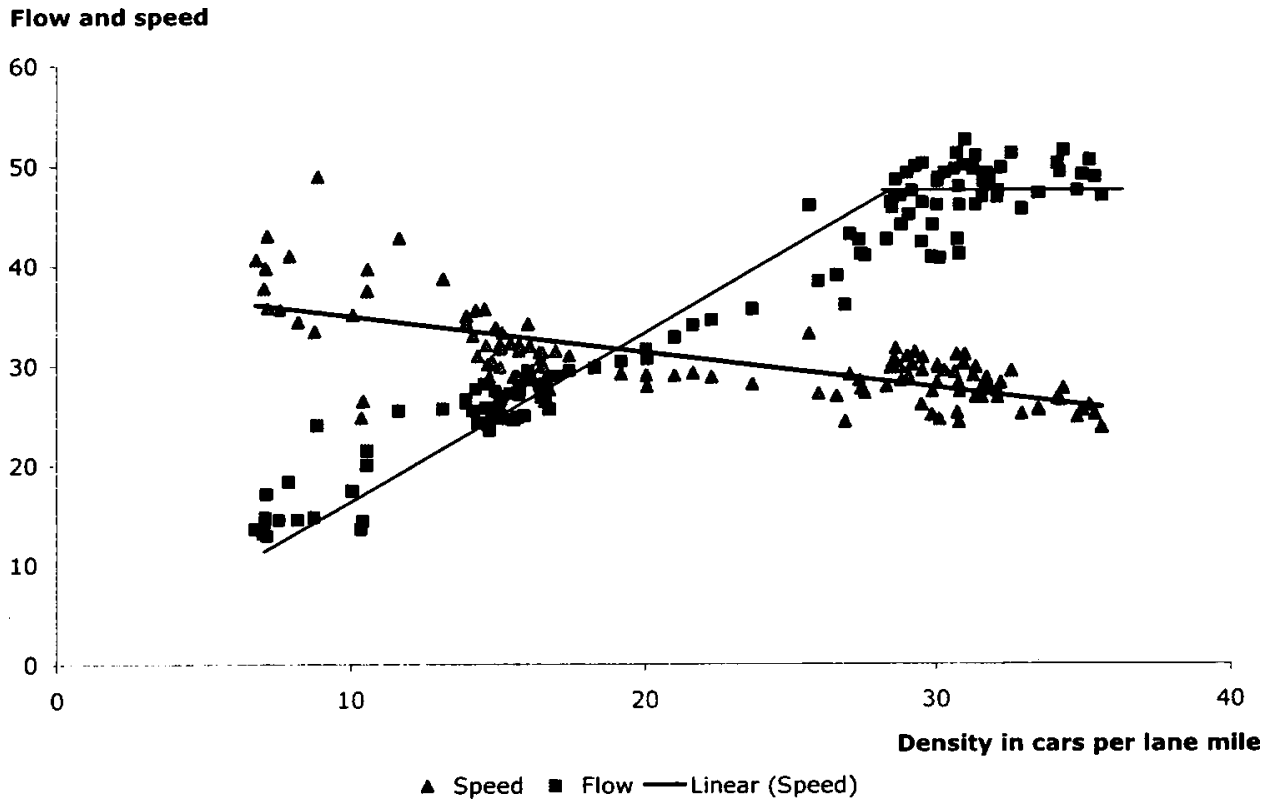

Fig. 9. Flow and speed varying by density on 83 (Dowling Avenue) 
If $\beta_{1}$ is not statistically different from zero, we have Phase 2 .

If we encounter a significant $\beta_{1}$ we know that it is not Phase 2 . Then we test the variation of speed over time.

\section{Phase 3a}

For a negative variation of speed over time, we test the variation of downstream flow over time and if this variation is positive, we conclude that higher flows are taking place downstream with time and the present section must be an active bottleneck. In this case, flow on the section drops and at the same time speed also drops, while flow downstream does not. Speeds drop to such an extent that vehicles cannot travel their own length in the service headway.

\section{Phase 3d}

For a negative variation of speed over time, both flow and speed drop on the section but flows downstream drop just before. This is indicated by the negative derivative of downstream flow with respect to time. Thus the present section is subject to an active bottleneck downstream.

\section{Phase 4}

Positive variation of section speeds with time indicates hysteresis and speed recovery. The identification typology is shown in Fig. 7 .

\section{Results}

\section{Phase 1: No Bottleneck Formation, neither Speeds nor Flows Drop}

Table 2 shows regression results of speed over density (Detector 52 near Broadway Avenue on Interstate 94 East Bound, November 3, 2000) which indicate that density is not a significant variable and so this location remains uncongested (Phase 1), where
Table 4. Phases and Phase-Switches for Three Representative Detectors

\begin{tabular}{|c|c|c|c|c|c|}
\hline \multirow[b]{2}{*}{ Detector } & \multirow[b]{2}{*}{ Time period } & \multicolumn{3}{|c|}{ Number of occurrences } & \multirow{2}{*}{$\begin{array}{l}\text { Number } \\
\text { of phase } \\
\text { switches }\end{array}$} \\
\hline & & Phase $3 \mathrm{a}$ & Phase $3 d$ & Phase 4 & \\
\hline 600 & 1405 to 1755 & 22 & - & 26 & 23 \\
\hline 83 & 1405 to 1755 & - & 24 & 24 & 23 \\
\hline 666 & 0530 to 1000 & 21 & 9 & 21 & 26 \\
\hline
\end{tabular}

${ }^{\mathrm{a}}$ Number of phase switches is the number of times the freeway section transitions from one phase to another, switches being random across time.

neither flow nor speed drops and no queuing or bottleneck formation/propagation takes place. This is corroborated by the flow-density relationship curve shown in Fig. 8.

\section{Phase 2: Speed Drops but Flow Does Not}

When it is not Phase 1 for a detector, we find the standard deviation of speeds for that detector and weed out the data for it for which speeds do not fall by at least one standard deviation. Then for the rest of the data, we find the statistical significance of density while regressing flow over density. Results for 83 on Interstate 94 (42nd Avenue) are shown in Table 3. We see the insignificance of density in this table and conclude that it is Phase 2 and speed should drop while flow remains almost the same. This is corroborated by the examining the speed-flow-density relationship in Fig. 9.

\section{Phases 3a, 3d, and 4}

Some detectors show a mix of Phases 3a, 3d, and 4. This indicates that, at the location of the detector, sometimes active bottleneck formation takes place, sometimes speed recovers and hysteresis takes place, and at other times, queue formation is a result of bottlenecks forming downstream. This is natural on a freeway with stochastic traffic flow. However often, only Phases $3 a$ and 4

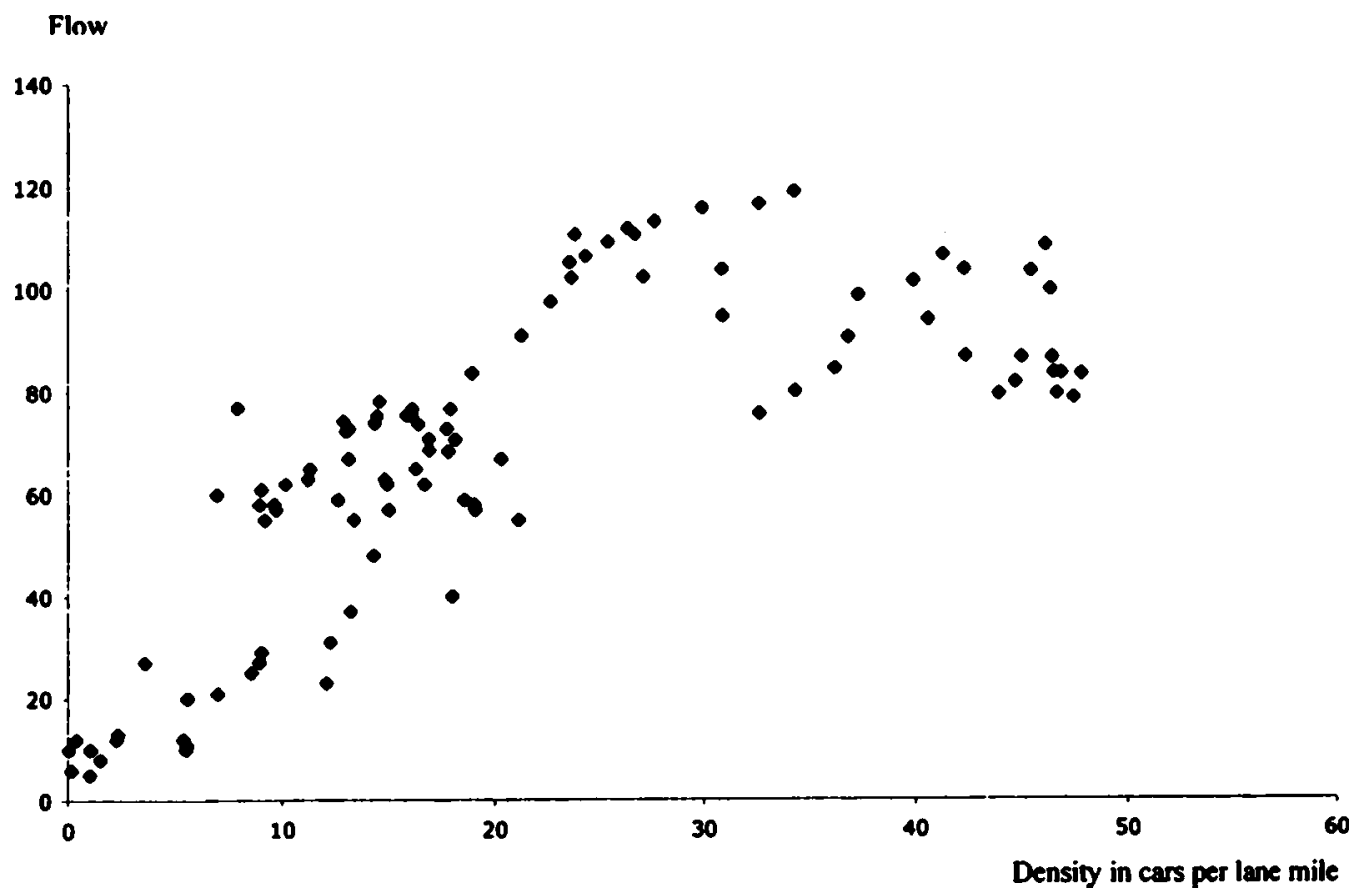

Fig. 10. Flow varying by density on 688 (49th/53rd Avenue) 
Table 5. Phases for Individual Sections on I-94 for the Period from 1 November, 2000 to 6 November, 2000

\begin{tabular}{|c|c|c|c|c|c|c|c|c|c|}
\hline \multirow[b]{2}{*}{ Date(s) } & \multicolumn{9}{|c|}{ Sections } \\
\hline & $\begin{array}{c}\text { Shingle Creek } \\
\text { Parkway }\end{array}$ & $\begin{array}{l}\text { Dupont } \\
\text { Avenue }\end{array}$ & 57th Avenue & $\begin{array}{l}\text { 49th/53rd } \\
\text { Avenue }\end{array}$ & $\begin{array}{c}\text { 42nd Avenue } \\
\text { 3a, 3d, } 4\end{array}$ & $\begin{array}{l}\text { Dowling } \\
\text { Avenue }\end{array}$ & $\begin{array}{l}\text { Lowry } \\
\text { Avenue }\end{array}$ & $\begin{array}{l}\text { Broadway } \\
\text { Avenue }\end{array}$ & $\begin{array}{c}\text { Plymouth } \\
\text { Avenue }\end{array}$ \\
\hline \multicolumn{10}{|l|}{ Morning peak } \\
\hline November 1, 2000 & $3 a, 4$ & $3 \mathrm{a}, 3 \mathrm{~d}, 4$ & 1 & 1 & $3 \mathrm{a}, 3 \mathrm{~d}, 4$ & 1 & 1 & 1 & $*$ \\
\hline November 2, 2000 & $3 \mathrm{a}, 3 \mathrm{~d}, 4$ & 1 & 1 & 1 & 1 & 1 & $3 \mathrm{~d}, 4$ & $3 \mathrm{a}, 3 \mathrm{~d}, 4$ & 2 \\
\hline November 3, 2000 & $3 a, 3 d, 4$ & 1 & 1 & 1 & 1 & 1 & 1 & $3 \mathrm{a}, 3 \mathrm{~d}, 4$ & 2 \\
\hline November 4, 2000 & 1 & 1 & 1 & $*$ & 1 & $3 \mathrm{~d}, 4$ & $3 \mathrm{~d}, 4$ & $3 \mathrm{a}, 3 \mathrm{~d}, 4$ & $*$ \\
\hline November 5, 2000 & $3 \mathrm{a}, 3 \mathrm{~d}, 4$ & $*$ & 1 & 1 & $*$ & $*$ & 1 & $*$ & $*$ \\
\hline November 6, 2000 & 1 & 1 & $3 \mathrm{~d}, 4$ & 3,5 & $3 \mathrm{~d}, 4$ & $3 \mathrm{~d}, 4$ & $3 \mathrm{~d}, 4$ & $*$ & $*$ \\
\hline \multicolumn{10}{|l|}{ Evening peak } \\
\hline November 1, 2000 & 2 & $3 a, 4$ & 1 & 1 & $3 \mathrm{~d}, 4$ & 1 & 1 & 2 & $*$ \\
\hline November 2, 2000 & 2 & 2 & 1 & 1 & 2 & 1 & 2 & 2 & 2 \\
\hline November 3, 2000 & $*$ & 2 & 2 & $3 \mathrm{a}, 3 \mathrm{~d}, 4$ & $3 a, 3 d, 4$ & $3 \mathrm{a}, 3 \mathrm{~d}, 4$ & 1 & 2 & 2 \\
\hline November 4, 2000 & 1 & 1 & $3 \mathrm{a}, 3 \mathrm{~d}, 4$ & $3 \mathrm{a}, 3 \mathrm{~d}, 4$ & $3 \mathrm{~d}, 4$ & $3 \mathrm{~d}, 4$ & $3 \mathrm{~d}, 4$ & $3 a, 4$ & 2 \\
\hline November 5, 2000 & $3 \mathrm{~d}, 4$ & 2 & 1 & 1 & 2 & $3 \mathrm{~d}, 4$ & 2 & 2 & $*$ \\
\hline November 6, 2000 & $3 \mathrm{~d}, 4$ & 2 & 1 & 1 & $3 \mathrm{~d}, 4$ & 1 & 1 & $*$ & $*$ \\
\hline
\end{tabular}

Note: The asterisked (*) fields are phases where either the detector was not functioning properly or the phase could not be ascertained due to nonavailability of data for downstream sections; and phases where only Phases $3 \mathrm{~d}$ and 4 occur and not $3 \mathrm{a}$ and the ones where Phases $3 \mathrm{a}$ and 4 occur but not $3 \mathrm{~d}$.

take place (or only Phases 3d and 4 take place) which suggest that the section may be considered an active bottleneck (or subject to downstream bottleneck). There are, however, instances where all three phases occur on one section as will be illustrated here. Table 4 shows a section on I-94 near Dupont Avenue, which fluctuates between the states of active bottleneck and recovery. It also shows a section defined by 83 (Dowling Avenue), which is largely affected by downstream bottleneck, but is itself not an active bottleneck. We can also see the third situation (Detector 666, Shingle Creek Parkway) where, for the early part of the morning peak, the section behaved mostly as an active bottleneck; though during the later part, a downstream bottleneck was affecting it. This example shows how all three phases can coexist for a single freeway section. There are a few occasions when none of the three phases appears. This is because those times are when it is either Phase 1 or 2. Any one section cannot be labeled as an "active bottleneck" in generic terms for this reason. The $Q-K$ relationship in Fig. 10 illustrates this. The summary table which shows the bottleneck properties for each section on Interstate 94 for the period from November 1 to November 6 is included in Table 5 .

\section{Summary and Conclusions}

This paper analyzes traffic flow parameters (flow, density, and speed) over various freeway sections on the Twin Cities freeway network. An algorithm is developed to balance flows on any freeway section ensuring flow conservation. This algorithm searches for the region of least error and then calibrates the other freeway section based on this least-error section. Errors in flows given by detectors have been minimized by the calibration procedure. Queuing analysis has been extensively applied to predict and track bottleneck formation and to detect active bottleneck locations. A typology was developed to determine the location of active bottlenecks on the freeways based on statistical analysis and their correspondence to visual relationships between flow, density, and speed, this typology helps us identify the locations that are prone to bottleneck formation and those that are prone to being affected by downstream bottlenecks. This typology was tested on data gathered from sections on Interstate 94. A sample of six days for I-94 for morning and evening peak periods shows that the same section cannot always be characterized as an "active bottleneck" location; at some times it is active and at others, it is subject to downstream bottlenecks. Traffic flow characteristics change and that leads to changing situations on each freeway section.

The methodology developed in this paper can be used to correct flawed loop detector data and find active bottleneck locations. Having done this, more intelligent ramp-metering algorithms can be developed that control ramp flows leading to such locations. This will improve freeway operations. As part of future work, more freeway sections can be examined over longer time periods.

\section{References}

Agyemang-Duah, K., and Hall, F. L. (1991). "Some issues regarding the numerical value of freeway capacity." Highway capacity and level of service, U. Brannolte, ed., Balkema, Rotterdam, 1-15.

Barbour, R., and Fricker, D. J. (1990). "Balancing link counts at nodes using a variety of criteria: An application in local area traffic assignment." Transportation Research Record, 1220, Transportation Research Board, National Research Council, Washington, D.C., 33-39.

Bertini, R. L. (2003). "Toward the systematic diagnosis of freeway bottleneck activation." IEEE 6th Annual Conf. on Intelligent Transportation Systems, Shanghai, China, IEEE, New York.

Cassidy, M. (2001). "Recent findings on simple attributes of freeway formation and propagation." IEEE Transportation Systems Conf. Proc., Oakland, Calif., IEEE, New York.

Cassidy, M., Anani, S., and Haigwood, J. (2002). "Study of freeway traffic near and off-ramp." Transp. Res., Part A: Policy Pract., 36(6), $563-572$.

Cassidy, M., and Bertini, R. (2001). "Some traffic features at freeway bottlenecks.” Transp. Res., Part B: Methodol., 33(1), 25-42.

Cassidy, M., and Chung, K. (2002). "Testing Daganzo's theory of multilane freeway traffic." California PATH working paper (UCB-ITSPWP-2002-9). 
Daganzo, C. (1997). Fundamentals of transportation and traffic operations, Pergamon, New York.

Jia, Z., Chen, C., Coifman, B., and Varaiya, P. (2001). "The PeMS algorithms for accurate, real-time estimates of g-factors and speeds from single-loop detectors." IEEE Transportation Systems Conf. Proc., Oakland, Calif., IEEE, New York.
Lawson, T., Lovell, D., and Daganzo, C. (1997). "Using the input-output diagram to determine the spatial and temporal extents of a queue upstream of a bottleneck." Transportation Research Record 1572, Transportation Research Board, Washington, D.C., 140-147.

Minnesota Department of Transportation (MDOT). (2002). 〈website http://www.dot. state.mn.us (May 2002). 